\title{
Molecular photoacoustic imaging using gold nanoparticles as a contrast agent
}

Chulhong Kim, Eun Chul Cho, Jingyi Chen, Kwang Hyun Song, Leslie Au, et al.

Chulhong Kim, Eun Chul Cho, Jingyi Chen, Kwang Hyun Song, Leslie Au, Christopher P. Favazza, Qiang Zhang, Claire M. Cobley, Younan Xia, Lihong V. Wang, "Molecular photoacoustic imaging using gold nanoparticles as a contrast agent," Proc. SPIE 7564, Photons Plus Ultrasound: Imaging and Sensing 2010, 75641V (25 February 2010); doi: 10.1117/12.843549 


\title{
Molecular photoacoustic imaging using gold nanoparticles as a contrast agent
}

\author{
Chulhong Kim ${ }^{1}$, Eun Chul Cho ${ }^{1}$, Jingyi Chen ${ }^{1}$, Kwang Hyun Song ${ }^{2}$, Leslie Au ${ }^{1}$, Christopher P. \\ Favazza ${ }^{1}$, Qiang Zhang ${ }^{1}$, Claire M. Cobley ${ }^{1}$, Younan Xia ${ }^{1}$, and Lihong V. Wang ${ }^{1 *}$ \\ ${ }^{1}$ Department of Biomedical Engineering, Washington University in St. Louis, Campus Box 1097, \\ One Brookings Dr. St. Louis, Missouri, 63130 \\ ${ }^{2}$ Division of Medical Physics \& Engineering, Department of Radiation Oncology, The University of \\ Texas Southwestern Medical Center at Dallas, 5323 Harry Hines Boulevard, Dallas, Texas 75390
}

*Corresponding author: 1hwang@,biomed.wustl.edu

\begin{abstract}
Gold nanoparticles have received much attention due to their potential diagnostic and therapeutic applications. Gold nanoparticles are attractive in many biomedical applications because of their biocompatibility, easily modifiable surfaces for targeting, lack of heavy metal toxicity, wide range of sizes $(35-100 \mathrm{~nm})$, tunable plasmonic resonance peak, encapsulated site-specific drug delivery, and strong optical absorption in the near-infrared regime. Specifically, due to their strong optical absorption, gold nanoparticles have been used as a contrast agent for molecular photoacoustic (PA) imaging of tumor. The plasmonic resonance peak of the gold nanocages (AuNCs) was tuned to the near-infrared region, and the ratio of the absorption cross-section to the extinction cross-section was approximately $\sim 70 \%$, as measured by PA sensing. We used PEGylated gold nanocages (PEG-AuNCs) as a passive targeting contrast agent on melanomas. After 6-h intravenous injection of PEG-AuNCs, PA amplitude was increased by $\sim 14 \%$. These results strongly suggest PA imaging paired with AuNCs is a promising diagnostic tool for early cancer detection.
\end{abstract}

Keywords: Photoacoustic tomography, molecular imaging, gold nanoparticles, B16 melanoma.

\section{INTRODUCTION}

Photoacoustic imaging is a hybrid biomedical imaging modality that can provide strong optical absorption contrasts with high ultrasonic spatial resolution. ${ }^{1,2}$ Since ultrasonic parameters determine the spatial resolution beyond one optical transport mean free path $(\sim 1 \mathrm{~mm})$ in PA imaging, the maximum imaging depth and resolution are scalable while diffusive photons are available.

The use of near-infrared (NIR) light is highly desirable to increase the penetration depth in optical imaging because of the weak optical absorption of hemoglobin and scattering of tissues in this spectral region. However, it is a challenge to assess tumors in deep tissues using only morphological and functional PA imaging based on intrinsic contrasts. Therefore, molecularly specific exogenous contrast agents are desired to enhance the sensitivity and specificity on tumor assessment using PA imaging in deep tissues. Organic dyes such as IRDye- 800 and indocyanine green have been used as contrast agents for PA imaging, ${ }^{3}$ but these small molecules suffer from rapid blood circulation time and relatively small optical absorption cross-sections. Recently, gold nanostructures ${ }^{4}$ and single-walled carbon nanotubes ${ }^{5}$ have been demonstrated as contrast agents for PA imaging in vivo. Practically, the toxicity of the carbon nanotubes is in question for in-vivo imaging. Gold nanostructures are promising candidates because of their strong light absorption in the NIR regime, bio-compatibility, and easy functionalization of surfaces with tumor recognition moieties. In this Proceeding, we demonstrate the use of PEG-AuNCs as a passive targeting contrast agent in PA imaging of B16 melanomas in vivo. ${ }^{6,7}$

Photons Plus Ultrasound: Imaging and Sensing 2010, edited by Alexander A. Oraevsky, Lihong V. Wang, Proc. of SPIE Vol. $7564,75641 \mathrm{~V} \cdot$ (C) 2010 SPIE · CCC code: 1605-7422/10/\$18 - doi: 10.1117/12.843549 


\section{METHODS AND MATERIALS}

A photograph of a dark-field confocal photoacoustic imaging system is shown in Figure 2. A Ti:sapphire laser (LT2211A, LOTIS TII) pumped by a Q-switched Nd:YAG laser (LS-2137, LOTIS) with 6-ns pulse duration and 10-Hz pulse repetition rate was used. Formed by a spherical conical lens and an optical condenser, the donut-shaped light illumination was coaxially aligned with the ultrasound focus in water. Dark-field confocal configuration provided a greater penetration depth and higher SNR. The light fluences on the skin were less than $6.9 \mathrm{~mJ} / \mathrm{cm}^{2}$, within the ANSI limit. A 10-MHz single-element ultrasound transducer was used as a detector. The axial and transverse resolutions are $125 \mu \mathrm{m}$ and $140 \mu \mathrm{m}$, respectively. By measuring PA amplitudes according to their arrival times, one-dimensional depthresolved image could be acquired, referred to as an A-line. Additional raster scanning along the one transverse direction enables the formation of two-dimensional depth-resolved images (B-scans), and further raster scanning along another traverse direction provides three-dimensional images of optical absorption heterogeneities in biological tissues. The acquired three-dimensional raw data can be processed in two forms: a maximum amplitude projection (MAP), a projection of the maximum PA amplitude along each A-line onto the corresponding plane, and a true three-dimensional image using Volview software (Kitware).

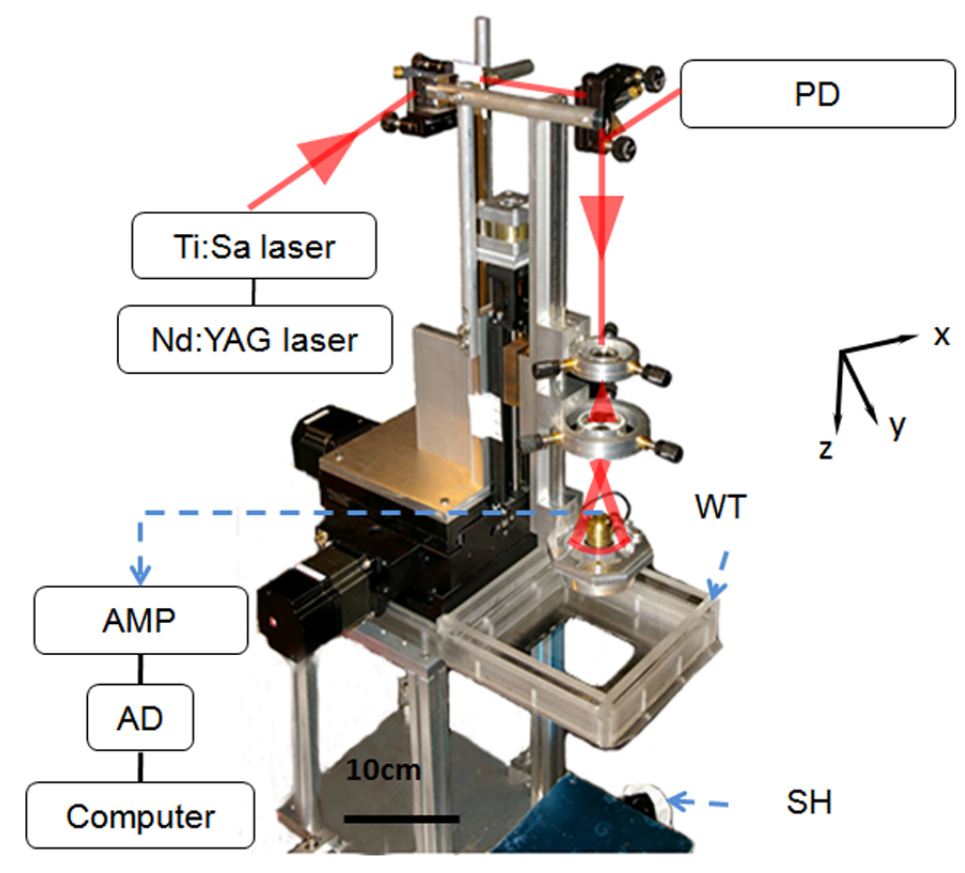

Figure 1: Photograph of volumetric photoacoustic imaging system. Ti:Sa laser; Ti:Sapphire laser, AMP; amplifier, AD; analog digital converter, PD; photodiode, WT; water tank, SH; sample holder.

Nude mice weighing about $30 \mathrm{~g}$ were used for the in-vivo experiments. All in vivo animal experiments were carried out in compliance with the Washington University Institutional Animal Care and Use Committee. The mice were initially anesthetized with a mixture of ketamine $(85 \mathrm{mg} / \mathrm{kg})$ and xylazine $(15 \mathrm{mg} / \mathrm{kg})$. During the PA imaging experiments, anesthesia was maintained using vaporized isoflurane ( $1 \mathrm{~L} / \mathrm{min}$ oxygen and $0.75 \%$ isoflurane, Euthanex Corp.), and vitals were monitored using a pulse oximeter (NONIN Medical INC., 8600V). The body temperatures of mice were maintained by a water heating pad.

\section{RESULTS AND DISCUSSIONS}

We investigated the feasibility of PA imaging of B16 melanomas in nude mice using PEG-AuNCs (Fig. 2). Prior to injection, a control image was acquired. Then, $100 \mu \mathrm{l}$ of PEG-AuNCs was intravenously injected to mice via the 
tailvein at concentration of $10 \mathrm{nM}$. A series of noninvasive volumetric PA images were obtained up to $6 \mathrm{~h}$ after injection in vivo. We could clearly see the enhancement of PA amplitude as time elapsed (Fig. 2).

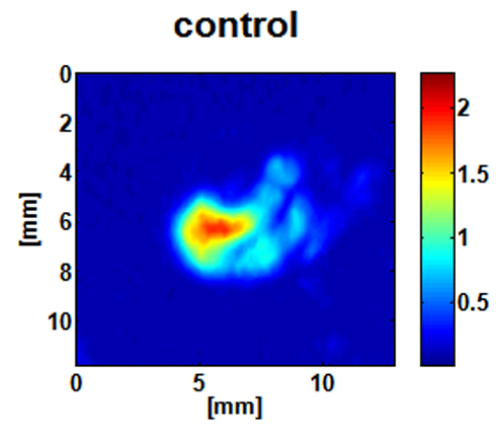

$1 \mathrm{hr}$ post injection

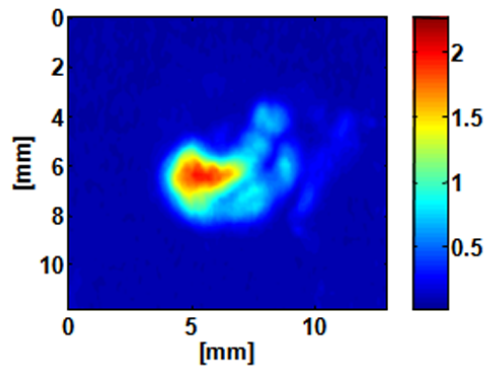

$6 \mathrm{hr}$

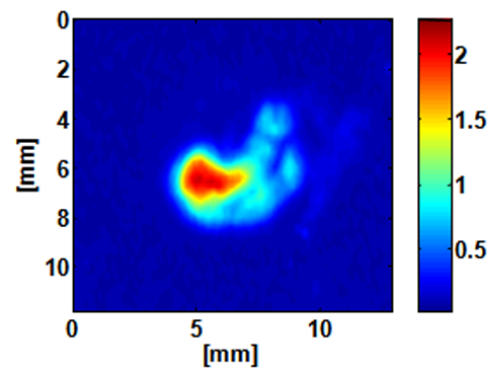

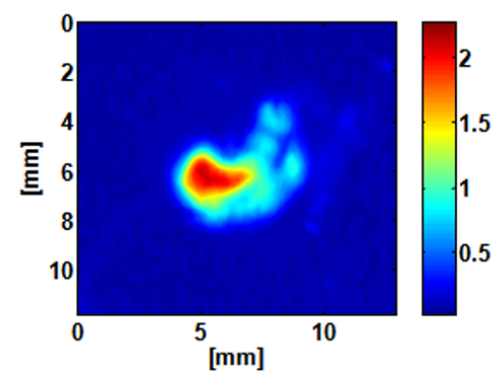

$3 \mathrm{hr}$
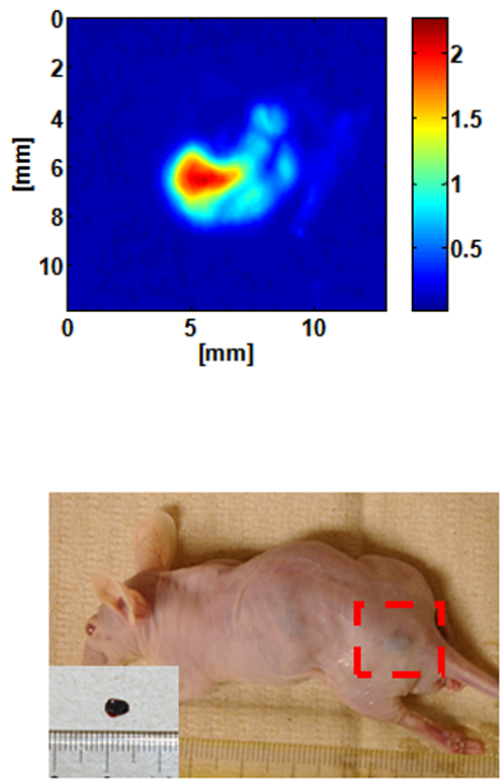

Figure 2: In vivo noninvasive PA MAP images of a B16 melanoma over time before and after injection of PEG-AuNCs

Figure 3 shows B-scan PA images from the same position of the animal before and after injection. The location of the melanoma was maintained.

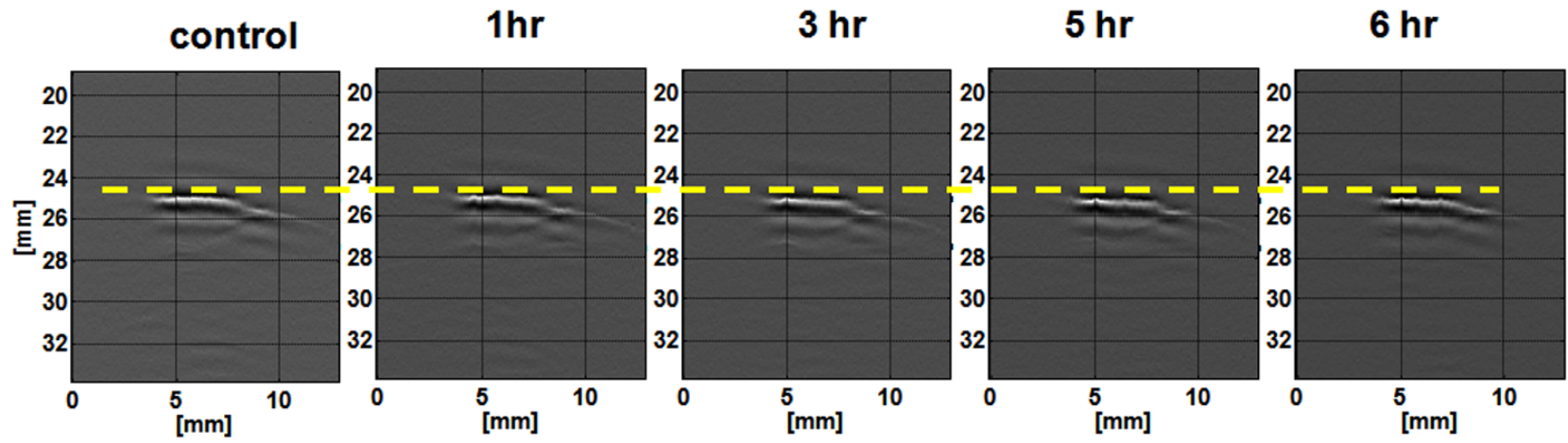

Figure 3: B-scan PA images of melanomas before and after injection of PEG-AuNCs.

Figure 4 shows plots for the enhancement of PA signals in the tumors over a period of time. The PA signals with PEGAuNCs show a monotonous increase untill $5 \mathrm{~h}$ post-injection and reached a level-off. The PA amplitude increased up to $14 \%$ at $6 \mathrm{~h}$ post-injection. 


\section{Signal at tumor area}

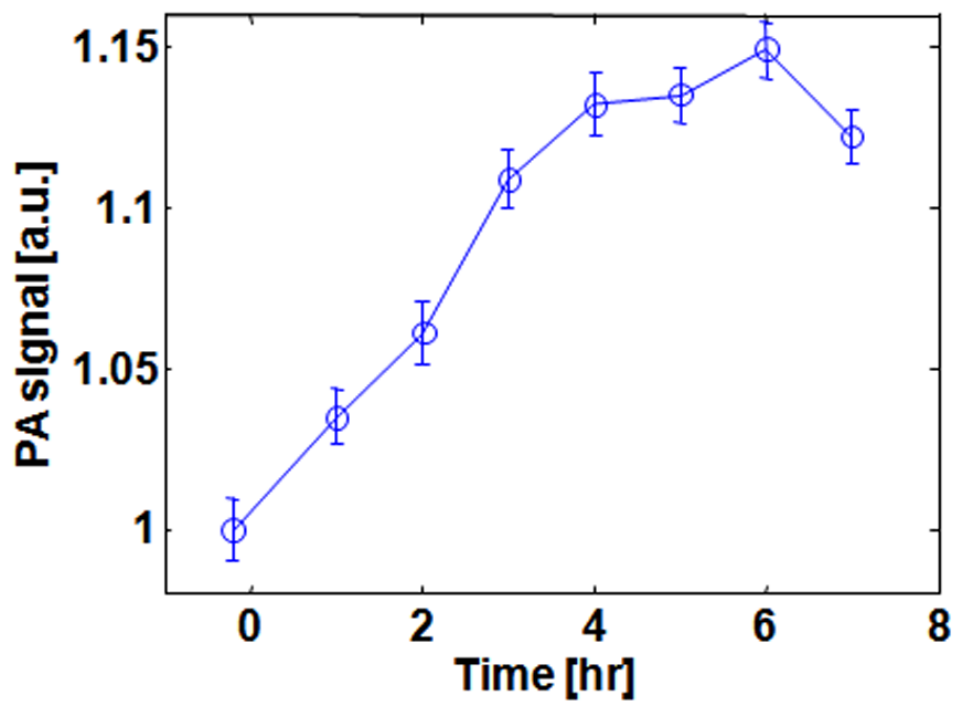

Figure 4: Increase in PA amplitudes within melanomas after intravenous injection of PEG-AuNCs.

\section{CONCLUSIONS}

In summary, we have demonstrated that passive targeting PEG-AuNCs can be used as a PA contrast agent on B16 melanomas in vivo. The detection sensitivity of the PA imaging system was $\sim 4.5 \mathrm{pM}$. Biocompatible AuNCs can be used as a diagnostic contrast agent for molecular PA imaging, a photothermal agent based on strong optical absorption, and a drug-delivery platform based on a hollow feature. Moreover, the circulation time of PEG-AuNCs in the blood stream is relatively long compared to organic dyes, which is an additional benefit for in vivo imaging. ${ }^{12}$ Potentially, high-resolution 3D morphological and functional PA imaging, when combined with AuNCs can diagnose tumors at early stages, treat tumors using either the photothemal effect or drug-delivery, monitor post-treatment processes, and further improve post-tumor treatment plans in real clinical application.

\section{ACKNOWLEDGEMENT}

This work was supported in part by grants from National Institutes of Health (R01 EB000712, R01 EB008085, and U54 CA136398 -- the Network for Translational Research -- to L.V.W.). L.V.W. has a financial interest in Microphotoacoustics, Inc. and Endra, Inc., which, however, did not support this work. Y.X. thanks the National Institutes of Health for a 2006 Director's Pioneer Award (DP1 OD000798-04). E.C.C. was also partially supported by a fellowship award from the Korea Research Foundation (KRF-2007-357-D00070) funded by the Korean Government. J.C. was supported by a Pilot Grant from Washington University Molecular Imaging Center.

\section{REFERENCES}

[1] X. D. Wang, et al., "Noninvasive laser-induced photoacoustic tomography for structural and functional in vivo imaging of the brain," Nat Biotechnol 21, 803-806 (2003).

[2] H. F. Zhang, K. Maslov, G. Stoica, and L. V. Wang, "Functional photoacoustic microscopy for high-resolution and noninvasive in vivo imaging," Nat Biotechnol. 24(7), 848-851 (2006).

[3] M. L. Li, et al., "Simultaneous molecular and hypoxia imaging of brain tumors in vivo using spectroscopic photoacoustic tomography," P IEEE 96, 481-489 (2008).

[4] P.C. Li, et al., "In vivo Photoacoustic Molecular Imaging with Simultaneous Multiple Selective Targeting Using Antibody-Conjugated Gold Nanorods," Optics Express 16, 18605-18615 (2008). 
[5] A. De la Zerda, et al. "Carbon nanotubes as photoacoustic molecular imaging agents in living mice," Nat Nanotechnol 3, 557-562 (2008).

[6] J. Y. Chen, et al,. "Gold nanocages: Engineering their structure for biomedical applications," Adv Mater 17, 2255 2261 (2005).

[7] S. E. Skrabalak, L. Au, X. D. Li, and Y. Xia, "Facile synthesis of Ag nanocubes and Au nanocages," Nat Protoc 2, 2182-2190 (2007). 